ACTA UNIVERSITATIS LODZIENSIS

Folia Litteraria Romanica 16, 2021

https://doi.org/10.18778/1505-9065.16.05

\author{
Anna Bochnakowa \\ Université Jagellonne de Cracovie \\ (iD) https://orcid.org/0000-0003-2707-3261 \\ anna.bochnak@uj.edu.pl
}

\title{
Les mots amour et miłość, aimer et kochać dans la langue française et polonaise : aspect sémantique, dérivation, phraséologie
}

\begin{abstract}
RÉSUMÉ
Ce texte est une présentation du fonctionnement des mots amour/miłość i aimer/kochać en français et en polonais, afin d'índiquer les différences de sens et d'emploi dans les deux langues. On compare des relations sémantiques entre les mots amour/miłość et amitié/przyjaźń, ainsi que leur expressivité. On présente aussi les dérivés, les phraséologismes contenant ces mots. Il est à constater une grande richesse synonymique du français aimer, ainsi qu'une différence sémantique entre les verbes polonais kochać et lubić et leurs équivalents français aimer et aimer bien/beaucoup renforcés par des adverbes d'intensité qui, paradoxalement, réduisent le sens émotionnel du verbe aimer 'aimer d'amour' au degré correspondant à lubić 'aimer bien' dans certains contextes. Bien que les mots amour et aimer appartiennent aux universaux du langage, leurs champs sémantiques diffèrent dans les deux langues.
\end{abstract}

MOTS-CLÉS - amour, aimer, sémantique, morphologie, langue française, langue polonaise

The Words amour and mitość, aimer and kochać in French and Polish: Semantics, Derivation, Phraseology

\section{SUMMARY}

The text is a presentation of the presence of the words amour/miłość (Eng. love) and aimer/kochać (Eng. to love) in French and Polish, taking into account the differences in semantics and use in both languages. The semantic relationship of the words amour/miłość and amitié/przyjaźń (Eng. friendship) is indicated, as well as the difference in the degree of semantic intensity between these words. Their derivative phrases and phraseological expressions in which they appear are also presented. The richness of the synonyms for the French word aimer is noted as well as the difference in the meaning of Polish words kochać and lubić (Eng. to like) and their French counterparts aimer and aimer bien/ beaucoup with the use of strengthening adverbs, which paradoxically reduce the intensity of the emotional meaning of the verb aimer 'to love' to a degree corresponding to the meaning of Polish lubic in specific contexts. Although the words amour and aimer belong to language universals, their meanings and scope of use turn out to be different in both languages.

KEYWORDS - love, to love, semantics, morphology, French language, Polish language 
La matière que nous traitons ici est belle, envoûtante, mais délicate, fragile et certainement vaste. Et si nous tentons de cerner le sens des mots amour et aimer, la tâche s'avère compliquée. Notre grand poète polonais Adam Mickiewicz a saisi le problème essentiel, celui de l'identification du sentiment dans le poème Niepewność (Incertitude) : «I znowu sobie zadaje pytanie : Czy to jest przyjaźń? Czy to jest kochanie? » ('Et encore une fois, je me pose cette question : est-ce de l'amitié ou de l'amour?').

L'incertitude du poète se concentre sur des sentiments proches, mais mis en opposition. En polonais, les mots amitié et amour ont des sources distinctes : przyjaźn provient d'une forme pré-slave qui constitue la racine de mots dans plusieurs langues de ce groupe, et reste en rapport avec le verbe sprzyjać 'accorder des faveurs, appuyer, encourager, aider' (Boryś, 2005 : 498). Mickiewicz a employé le mot kochanie 'action d'aimer', synonyme de miłość 'amour'. Le verbe courant pour aimer est kochać, du pré-slave dialectal *kochati, avec une variante *kosati 'toucher, caresser', mais aussi 'soigner, faire plaisir à quelqu'un, manifester de la bienveillance, aimer' (Boryś, 2005 : 241).

En français, amour et amitié remontent respectivement aux mots latins amor et amicitia, qui ont un tronc commun. Malgré la ressemblance formelle, le sens des deux mots reste bien distinct. Tout comme Mickiewicz, l'auteur anonyme de la citation connue suivante met en opposition les deux notions : « Un jour, l'amour a dit à l'amitié : Pourquoi existes-tu, puisque je suis là ? L'amitié lui répond: Pour amener un sourire là où tu as laissé des larmes ».

\section{Amitié et amour, przyjaźń et mitość dans les dictionnaires français et polonais}

Quand on parcourt l'article amitié dans le TLF informatisé, on y trouve plusieurs citations littéraires soulevant l'opposition entre les deux sentiments, ainsi que la valeur sémantique du mot amitié dans des collocations ou des synonymes; forme de sympathie, association de complaisance mutuelle, amitié profonde des cours et des esprits, une complicité, amitié féminine, amitié masculine, tendre et constante amitié, ancienne, fidèle amitié, visite d'amitié, des amitiés particulières, etc.

Le TLFi donne aussi quelques locutions : en toute amitié ; par amitié pour ; avoir, éprouver de l'amitié pour ; transmettre, envoyer ses amitiés. Nous renonçons à l'énumération des adjectifs qui peuvent qualifier le mot amitié, ils sont nombreux.

Pour le polonais, nous avons consulté Wielki słownikjęzyka polskiego, le grand dictionnaire du polonais contemporain, publié uniquement en ligne et toujours en cours d'élaboration. Nous constatons un parallélisme dans la définition et le fonctionnement lexical du mot przyjaźn avec le français amitié, comme dans le cas des collocations avec les adjectifs dtugoletnia ('longue'), serdeczna ('cordiale'), szczera ('sincère'), etc. Nous citerons seulement deux détails contredisant cela : 
l'expression szorstka przyjaźń 'une amitié dure, rude', littéralement 'rugueuse' pour décrire une relation entre hommes politiques rivaux qui tiennent à garder les apparences d'une certaine entente, et l'adjectif przyjazny 'amical' employé abusivement par rapport aux objets, comme par exemple słownik przyjazny użytkownikom pour louer les qualités d'un dictionnaire et qui ressemble un peu à l'utilisation de conviviale (s) dans lunettes, voitures, coiffures, installations conviviales, utilisation ridiculisée dans une série d'articles consacrés à la langue dans Le Figaro d'il y a quelques années... (Bochnakowa, 2005 : 48-49).

Quant à l'amour, sentiment universel que nous ne tenterons pas de définir ici, nous nous limitons à l'aspect lexical du mot dans plusieurs acceptions. Ainsi, le TLFi évoque-t-il l'emploi du mot dans le sens de 'communion intime avec l'univers, aussi par rapport à Dieu', « Dieu est amour »; le dictionnaire fournit des locutions : pur amour, pour l'amour de Dieu, aussi utilisée comme exclamation ; l'amour comme principe de cohésion de la société : l'amour de l'humanité, l'amour de la patrie, l'amour entre personnes l'amour familial, maternel, paternel, filial, fraternel, conjugal, enfin l'amour comme lien passionnel entre deux personnes, avec des spécifications : l'amour physique, platonique, unique, romantique, incestueux, l'amour homosexuel, le grand amour, l'amour parfait, l'amour désintéressé, impossible, l'amour malheureux, l'amour libre, l'amour courtois - avec ses connotations dans l'histoire littéraire, l'amour charnel, fou, interdit, etc. Et enfin l'amour propre qui ne demande pas de partenaire et qui prend un sens particulier. On parle d'amour de la nature, de l'argent, de la musique et de tout ce qui peut être une passion ou un intérêt de l'homme. Il faut citer aussi la tendre formule d'adresse mon amour et le terme d'art un amour comme personnification de l'amour par un enfant rondelet.

Le mot au pluriel signifie, ou bien le sentiment dans ses différentes étapes, ou bien des liens successifs avec des personnes différentes. Et en levant un toast en disant : à tes amours, on peut penser aux deux.

Le mot amour complète parfois des substantifs comme chagrin d'amour, lettre d'amour, acte d'amour, nid d'amour, signe d'amour, jeu d'amour, plaisir d'amour, viole d'amour 'instrument de musique'; enfant de l'amour peut désigner un enfant naturel ou un enfant désiré et conçu comme fruit d'un amour profond Et enfin, citons quelques proverbes et dictons concernent l'amour, à commencer par une affirmation ironique : L'amour du prochain commence par soi-même ou encore celui-ci, élevant l'amour à un facteur vital mais se rapportant à une situation matérielle difficile : vivre d'amour et d'eau fraîche; l'amour semble pourtant récompenser un manque ou une perte de ressources : malheureux au jeu, heureux en amour, froides mains - chaudes amours. Sans avoir épuisé la richesse d'emploi du mot amour, nous tenons encore à rappeler ses dérivés : amoureux, substantif et adjectif, l'adverbe amoureusement, le diminutif amourette pour une liaison passagère, ou comme nom de plante, ou bien encore, avec une majuscule, en tant que nom d'un modèle de soutien-gorge de la marque allemande Triumph. 
Au pluriel, le mot a son emploi culinaire : 'moelle épinière de veau ou de mouton servie comme garniture' ou encore 'rognons blancs', c'est-à-dire les testicules de jeunes béliers, de verrats ou de taureaux, terme utilisé surtout en français canadien. Notons encore un dérivé verbal quelque peu péjoratif s'amouracher.

En polonais, le mot miłość 'amour' provient du pré-slave reconstitué *milostb (Boryś, 2005 : 329), et reste en rapport avec l'adjectif mity 'agréable, bienveillant, plaisant'. Comme le français amour, il apparaît dans plusieurs nuances sémantiques et emplois qui sont des équivalents du mot français. Dans le répertoire que nous avons présenté pour le français, on trouve pourtant des expressions qui ne fonctionnent pas en polonais : amour courtois a son équivalent littéral miłość $d$ worska, mais il n'est pas doté du poids culturel propre à l'usage en français ; amour n'est pas tellement évoqué dans des toasts, c'est la santé qui y est appelée. L'amour personnifié dans la peinture ou dans la sculpture est désigné en polonais avec le diminutif amorek, provenant du mot latin. Miłość 'amour' figurait dans l'ancienne formule d'adresse wasza miłość littéralement 'votre amour', utilisée autrefois par rapport au roi ou aux personnes haut placées. Le terme, fréquent dans le discours, s'est réduit à waszmość et puis même à waść, dans lesquels l'élément miłość se trouve totalement effacé.

Parmi les expressions phraséologiques, Wielki słownik języka polskiego note miłość jego/jej życia 'l'amour de la vie de quelqu'un', mitość od pierwszego wejrzenia 'coup de foudre', littéralement 'amour dès le premier regard', cywilizacja miłości 'civilisation de l'amour', notion introduite dans la langue de l'Église par le pape Paul VI et développée par Jean Paul II, aujourd'hui souvent citée par les théologiens catholiques par opposition à la civilisation de la mort incarnée par l'avortement, l'euthanasie, le génocide.

Quant aux dérivés, le principe de formation n'est pas le même qu'en français : amoureux se traduit par zakochany, du verbe zakochać się 'tomber amoureux', ou bien, littéralement par milosny, adjectif de relation.

Amourette au sens propre à son équivalent de sens milostka et aussi sa forme diminutive en polonais. Amourettes comme plat n'a pas de nom aussi tendre, mais reste un terme d'anatomie animale. Notons encore que le dérivé verbal français s'amouracher n'a pas d'équivalent en polonais standard, seulement un verbe familier et quelque peu dépassé (za)bujać się w kimśs 'tomber amoureux, être amoureux', dans le sens figuré du verbe signifiant '(se) balancer, tanguer, voler dans l'air'. Bujać comme verbe transitif veut dire familièrement 'mentir à quelqu'un, le tromper'.

Le répertoire des proverbes et des dictons polonais ne contient pas celui sur les mains froides ni sur l'amour et l'eau fraîche comme moyens de subsister, mais il en existe un, très optimiste : Stara/pierwsza miłość nie rdzewieje 'Le vieil/le premier amour ne rouille pas'. L'amour est souvent évoqué dans les manifestations de la sagesse populaire, et les proverbes y faisant référence se multiplient aussi bien en français qu'en polonais, nous nous permettons de citer quelques dictons polonais, 
parfois anciens et oubliés, trouvés dans Księga przystów polskich (Adalberg, 1889-1894 : 308-310) Mitość i kaszel nigdy się nie ukryja 'L'amour et la toux ne passent jamais inaperçus', Miłość mężczyźnie wchodzi przez oczy, a kobiecie przez uszy 'L'amour s'infiltre dans un homme par les yeux, et dans une femme par les oreilles' ; Jak mitość oknem zajrzy, to bieda drzwiami włazi 'Quand l'amour regarde par la fenêtre, la misère s'introduit par la porte' ; Miłość własna nie ma żadnego przyjaciela 'L'amour propre n'a aucun ami'. Et le recueil d'Adalberg cite ainsi des dizaines d'expressions avec le mot miłość.

\section{Aimer et kochać}

Aimer dans son sens absolu appelle ses quasi-synonymes dans la langue standard : adorer, chérir, être amoureux, avoir quelqu'un dans la peau, porter dans son cour, raffoler. Ces verbes forcément transitifs appellent des compléments variés et désignent le sentiment d'amour à différent degré. L'action d'aimer peut avoir comme objet Dieu, la famille et les proches, une personne particulière à laquelle on voue un fort sentiment d'attachement. On peut aimer les animaux en tant qu'êtres proches et personnalisés, mais la qualité du sentiment se réalise autrement. Le verbe aimer peut prendre comme complément aussi un substantif non animé de nature diverse : la patrie, la nature, l'art, le chocolat, le cinéma, etc., mais il porte une nuance de qualité et d'intensité. Aimer peut avoir comme complément, direct ou indirect, un verbe, et son sens se voit souvent modifié : aimer faire quelque chose exprime plutôt une prédilection ; un désir ou une volonté, s'il est au conditionnel j'aimerais partir; dans la construction indirecte : aimer à, aimer de cela exprime plus un choix, une préférence que le sentiment, et il prend le sens d'aimer bien, aimer beaucoup. Ces dernières locutions sont intéressantes, car l'adverbe de sens positif, diminue la valeur sémantique du verbe et devient synonyme de préférer, apprécier, trouver bon, beau, valable, plaisant et peut appeler un objet animé ou non animé et se rapproche par sa signification d'aimer le chocolat, le cinéma, etc. où le verbe perd ses connotations d'amour 'sentiment, passion amoureuse'. La même nuance revient au verbe raffoler de 'aimer beaucoup'.

Chérir est plutôt littéraire aussi bien dans le sens 'd'aimer d'amour (d'où mon chéri), que d'aimer bien. Adorer, primitivement lié au latin orare 'prier' avait la connotation religieuse de adorer une divinité, Dieu, 'vénérer, lui vouer un culte' passe dans un emploi plus large, synonyme emphatique d'aimer.

La forme pronominale s'aimer a un sens de réciprocité et aussi celui de faire l'amour. Formellement, cette locution se range à côté de faire la cuisine ou faire les courses, ce qui peut rendre perplexe quand on considère leur sens, mais reste un trait spécifique du système verbal du français, recourant souvent à ce type de nomination des actions. 
Le polonais kochać est équivalent du français aimer dans le sens large. Kocham Andrzeja correspond exactement à j'aime André, avec tout le poids du verbe. Mais le français j'aime le chocolat ou le cinéma sera traduit par lubię czekoladę, lubię kino, car le polonais dispose d'un verbe simple lubić venant du pré-slave *ljubiti (Boryś 2005 : 290), correspondant sémantiquement à aimer bien, aimer beaucoup. L'emploi de kochać par rapport au chocolat ou au cinéma est possible, mais emphatique, exalté, vu la différence de sens nettement sentie entre lubić et kochać. Lubić apparaît dans plusieurs collocations, le WSJP suggère les exemples suivants : lubić studentów 'aimer (bien) ses étudiants' lubić pić mleko, 'aimer boire du lait', lubić ciepło $i$ wilgoć, 'aimer la chaleur et l'humidité'. Le polonais, tout comme l'anglais to like et to love, diversifie la qualité du sentiment d'attachement, de préférence orienté vers son objet.

Le mot kochać a comme dérivés le substantif kochanie, synonyme d'amour utilisé par Mickiewicz ; kochanek 'amant', adjectif venant du participe passif kochany 'aimé' ; et une forme perfective et réfléchie : zakochać się correspond à tomber amoureux aussi bien de quelqu'un que par exemple de l'alpinisme.

Nous avons encore le verbe miłować (de miłość 'amour'), 'aimer', aujourd'hui ressenti comme soutenu, littéraire, archaïque, synonyme de kochać, surtout dans la langue d'église, pouvant être comparé pour sa valeur à chérir. Adorować, correspondant formel d'adorer lui équivaut seulement dans l'emploi religieux. Le sens courant est celui de faire la cour. La forme pronominale kochać się a deux sens, les mêmes qu'en français : la réciprocité et l'acte sexuel. Nous utilisons en revanche le verbe ubóstwiać, dérivé de bóstwo 'divinité' au sens proche d'idolâtrer, pour un emploi rappelant celui de adorer, raffoler.

À noter que le polonais manque l'équivalent de la belle expression française avoir quelqu'un dans la peau...

\section{Récapitulation}

Le champ lexical choisi comme sujet du colloque est très vaste et concerne des universaux sémantiques, il nous semble donc impossible de cerner le vocabulaire qui illustrerait la valeur des mots amour et aimer. Nous avons essayé de présenter leur fonctionnement sémantique en français et en polonais, et nous avons constaté, à côté des emplois parallèles, une richesse notable de synonymes du verbe aimer (et nous nous sommes limitée au français standard). En revanche, le polonais offre une différence de sens entre kochać et lubić que le français confond pour certains emplois ou bien qu'il exprime en complétant le verbe aimer par les adverbes bien et beaucoup, ce qui atténue et modifie le sens du verbe même.

Et nous tenons à terminer par une autre référence à Mickiewicz et à son poème Pan Tadeusz (Messire Thadée), considéré comme notre épopée nationale et qui raconte quelques jours dans la vie mouvementée d'une famille noble vivant dans 
un manoir traditionnel en Lituanie, juste avant et après le passage de l'armée de Napoléon en 1812. Le douzième et le dernier chapitre se terminant par un mariage est intitulé Kochajmy się! 'Aimons-nous' et, compte tenu du contenu de l'épopée, cette invitation dépasse le contexte conjugal et peut nous concerner tous.

\section{Bibliographie}

ADALBERG, Samuel (1889-1894), Księga przysłów, przypowieści i wyrażén przysłowiowych, Warszawa. (https:/www.wbc.poznan.pl/dlibra/publication/11603/edition/18873?language=pl, consulté le 20 janvier 2020)

BOCHNAKOWA, Anna (2005), Le bon français de la fin du XX eiècle. Chroniques du "Figaro » (1996-2000), Kraków, Wydawnictwo Uniwersytetu Jagiellońskiego

\section{Dictionnaires}

BORYŚ, Wiesław (2005), Słownik etymologiczny języka polskiego, Kraków, Wydawnictwo Literackie

Trésor de la langue française informatisé (http://atilf.atilf.fr/tlfv3.htm, consulté le 18 janvier 2020) Wielki stownik języka polskiego (https://www.wsjp.pl/, consulté le 15 janvier 2020)

Anna Bochnakowa - professeure de linguistique romane à l'Université Jagellonne de Cracovie. Domaines de recherche : lexicologie et lexicographie françaises et polonaises, contacts de langues, histoire du français. Auteure d'une centaine de publications, dont les monographies : Terminy kulinarne romańskiego pochodzenia $w$ języku polskim do końca XVIII wieku ; Le Nouveau grand dictionnaire françois, latin et polonois et sa place dans la lexicographie polonaise; Le bon français de la fin du XXe siècle. Chroniques du « Figaro » (1996-2000). 